\title{
Iliocaval stent reconstruction following inferior vena cava filter associated chronic thrombotic occlusion in a patient with factor $V$ leiden mutation - A case report and review of literature
}

\author{
Khan O Mohammad ${ }^{1}$, Jose BC Rodriguez ${ }^{2}$, Abdul M Zafar ${ }^{3}$ and Tariq Siddiqui ${ }^{2 *}$ \\ ${ }^{1}$ Paul L Foster School of Medicine, Texas Tech University Health Sciences Center, El Paso, Texas, USA \\ ${ }^{2}$ Division of Cardiovascular Medicine, Texas Tech University Health Sciences Center, El Paso, Texas, USA \\ ${ }^{3}$ Department of Radiology, Texas Tech University Health Sciences Center, El Paso, Texas, USA
}

\begin{abstract}
Iliocaval occlusion is an increasingly recognized complication following inferior vena cava (IVC) filter placement with an incidence ranging from 3-30\%. While there is evidence that this condition can be managed with endovascular stenting, there has been limited discussion regarding management of patients with iliocaval occlusion who also have hereditary thrombophilias. We describe a case of a 44-year-old male with Factor V Leiden mutation (FVL) who developed IVC filter-related post-thrombotic iliocaval occlusion and was treated using endovascular stenting. This case aims to highlight the utility of endovascular stenting in patients with thrombophilias who develop iliocaval occlusion and discuss the relevant literature.
\end{abstract}

\section{Introduction}

Patients with Factor V Leiden (FVL) mutation is at an increased risk for recurrent thrombotic events and may require placement of an inferior vena cava (IVC) filter to prevent pulmonary emboli. Paradoxically, IVC filters may cause recurrent deep vein thromboses (DVT) and related complications. Although available literature encompasses diverse venous pathologies, in general, IVC and Iliac vein stenting improve clinical outcomes in cases with venous outflow obstruction.

\section{Case series}

We present a case of a 44-year-old male with FVL, hypertension (HTN), and recurrent DVTs/pulmonary embolisms (PE) despite treatment with warfarin. He had an IVC filter in place for 9 years and did not have any acute thrombotic events since starting treatment with apixaban.

He presented with worsening bilateral lower extremity pain, edema, bilateral phlegmasia cerulea dolens and varicoceles. Estimated Clinical-Etiology-Anatomy-Pathophysiology (CEAP) classification at presentation was C4a. Lower extremity Doppler showed chronic nonocclusive right iliofemoropopliteal DVT and chronic non-occlusive left femoropopliteal DVT. CT abdomen and pelvis with intravenous contrast demonstrated extensive chronic thrombosis of the IVC below the IVC filter as well as bilateral common/external iliac veins with blood from bilateral common femoral veins draining through anterior abdominal wall collaterals into the anterior thoracic vein and recanalized umbilical vein (Figures 1 and 2).

Bilateral deep femoral veins were accessed at mid-thigh level using ultrasound guidance and micro-puncture needles (5F ministick $21 \mathrm{~g}$,
Vascular solution). Deep femoral venograms showed total occlusion of IVC below the filter and bilateral common iliac veins with extensive abdominopelvic collaterals. An angled NeviCross support catheter (Terumo Interventional System), multiple 0.014 wires Hi-Torque Command (Abbott), Hi-Torque Pilot (Abbott), Confianza pro wire

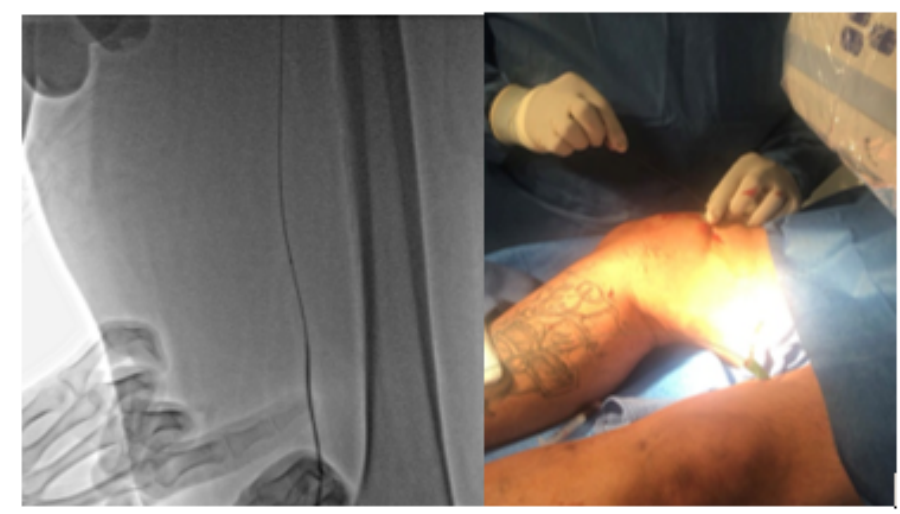

Figure 1. Right deep femoral vein access at mid-thigh level using micro puncture needle Bilateral access was obtained in the same fashion

${ }^{\star}$ Correspondence to: Tariq Siddiqui, Division of Cardiovascular Medicine, Texas Tech University Health Sciences, El Paso, 4800 Alberta Avenue, El Paso Texas 79905, United States, E-mail: Tariq.siddiqui@ttuhsc.edu

Key words: iliocaval occlusion, thrombophilia, stent, IVC filter

Received: August 06, 2020; Accepted: August 11, 2020; Published: August 21, 2020 
Mohammad KO (2020) Iliocaval stent reconstruction following inferior vena cava filter associated chronic thrombotic occlusion in a patient with factor V leiden mutation - A case report and review of literature

12g (ASAHI) and an 0.035 stiff glidewire (Terumo Interventional System) were used to cross the IVC occlusion. A Uni-fuse infusion catheter (AngioDynamics) was placed across the occlusion and tissue plasminogen activator (tPA) was infused for 24 hours for catheter directed thrombolysis (CDT).

On the second day, we attempted to cross the chronic left iliac occlusion using right IJV access. We able to cross into the proximal deep femoral vein with the support of an angled multipurpose catheter/NeviCross support catheter, similar as above 0.014 wire and 0.035 stiff glide wire. However, an attempt to advance distally was unsuccessful. The left iliac vein was sequentially pre-dilated with a $3.0 \times 20 \mathrm{~mm}$ Emerge balloon followed by $4.0 \times 20 \mathrm{~mm}$ noncompliant Emerge balloon. An 0.014 wire introduced through the right IJV was snared with a goose neck 5.0 introduced through the left femoral vein sheath. The 0.014 wire was exchanged for a 0.035 stiff glide wire. After placing bilateral femoral vein approach stiff glide wires across the IVC occlusion, sequential simultaneous pre-dilatations were performed from the IVC filter to the common femoral veins. Evaluation with Philips intravascular ultrasound (IVUS) catheter showed satisfactory improvement in luminal patency. Side-by-side Vici stents $(16 \times 120 \mathrm{~mm}$ and $16 \times 90 \mathrm{~mm}$ Boston scientific) were simultaneously deployed in the mid IVC. Bilateral Venovo stents $(16 \times 160 \mathrm{~mm}$, Bard) were deployed from inferior IVC to the bilateral common femoral veins and post dilated simultaneously with $14 \times 40 \mathrm{~mm}$ and $16 \times 40 \mathrm{~mm}$ Atlas balloons (Bard). Post deployment IVUS showed appropriate apposition of the stents with no dissection. Final bilateral iliocaval venograms showed patency of IVC and bilateral iliac veins with remarkable resolution of the abdominopelvic collaterals (Figures 3 and 4).

The patient reported sustained improvement in his symptoms at 24 hours and one month follow up. Estimated CEAP classification improved to $\mathrm{C} 2$ at one-month follow-up.

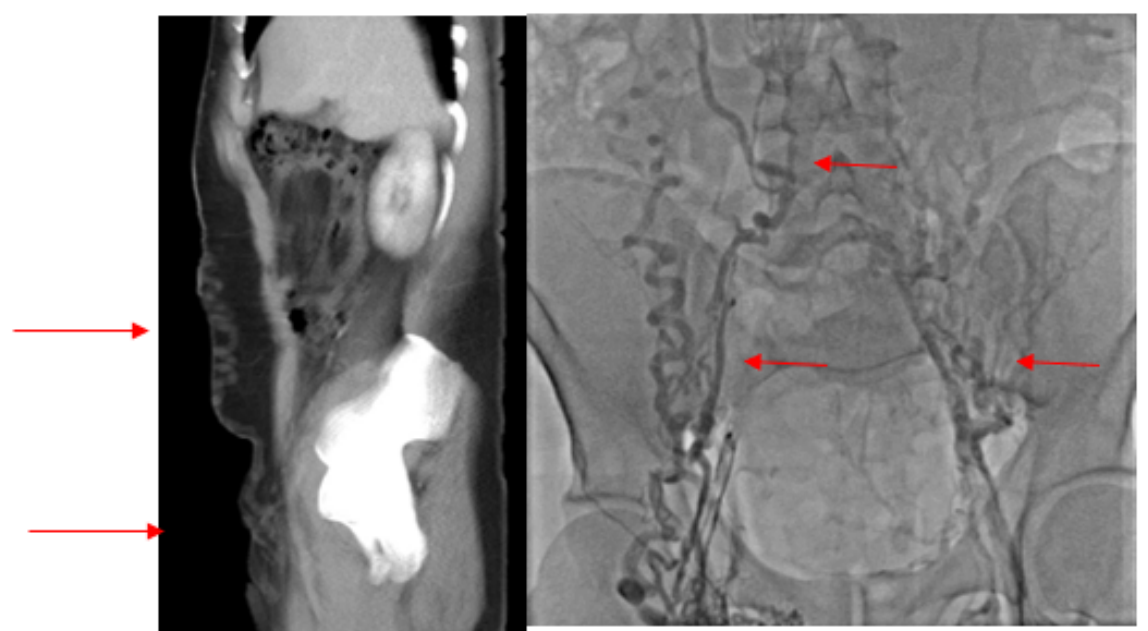

Figure 2a and 2b. CT with venous phase and initial bilateral venogram showing extensive pelvic and abdominal collaterals and complete IVC and bilateral iliac vein occlusion
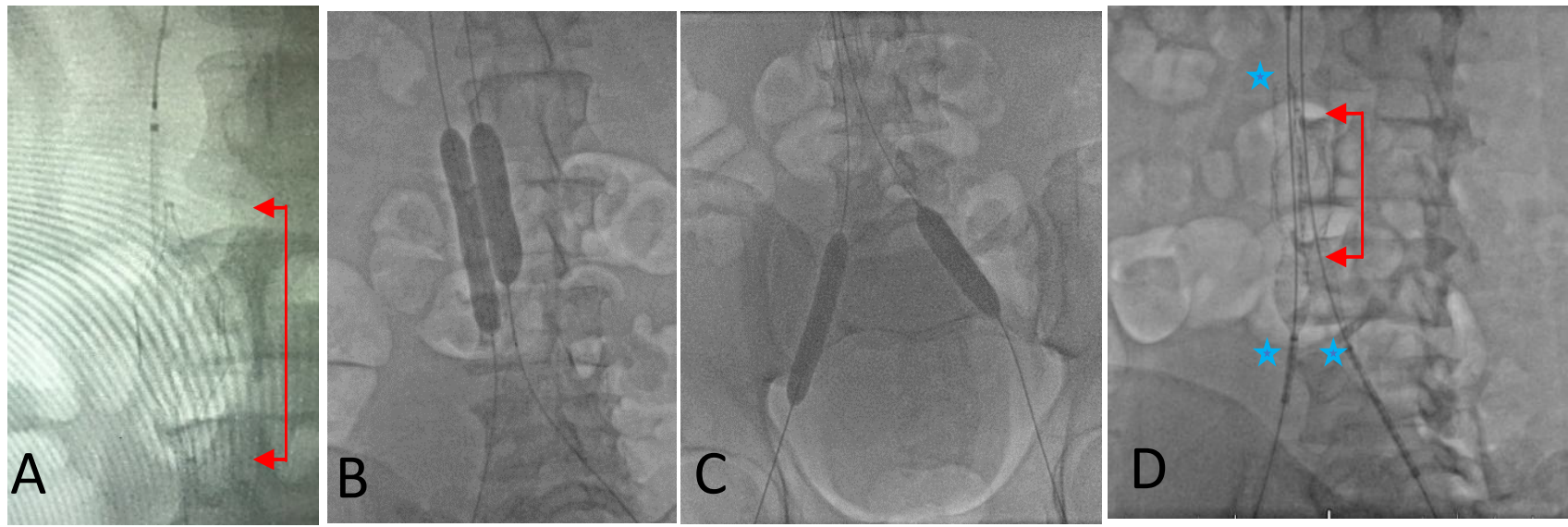

Figure 3. A. Final position of Unifuse catheter across the IVC filter (arrows) on day 1. Left in position for 24 hours. B and C. Sequential simultaneous predilatations was performed with $3.0 \times 150 \mathrm{~mm}, 6.0 \times 200 \mathrm{~mm}, 10 \times 60 \mathrm{~mm}$ and $12 \times 40 \mathrm{~mm}$ Evercross balloon. At the iliac bifurcation level, we simultaneously predilated with Atlas balloon $14 \times 40 \mathrm{~mm}$ on right and $16 \times 40 \mathrm{~mm}$ on the left. D. Vici stents $16 \times 120 \mathrm{~mm}$ and $16 \times 90 \mathrm{~mm}$ on either side of the center of the IVC filter (arrows). Stars mark the origin of the stent above the filter and sites of simultaneous deployment. 


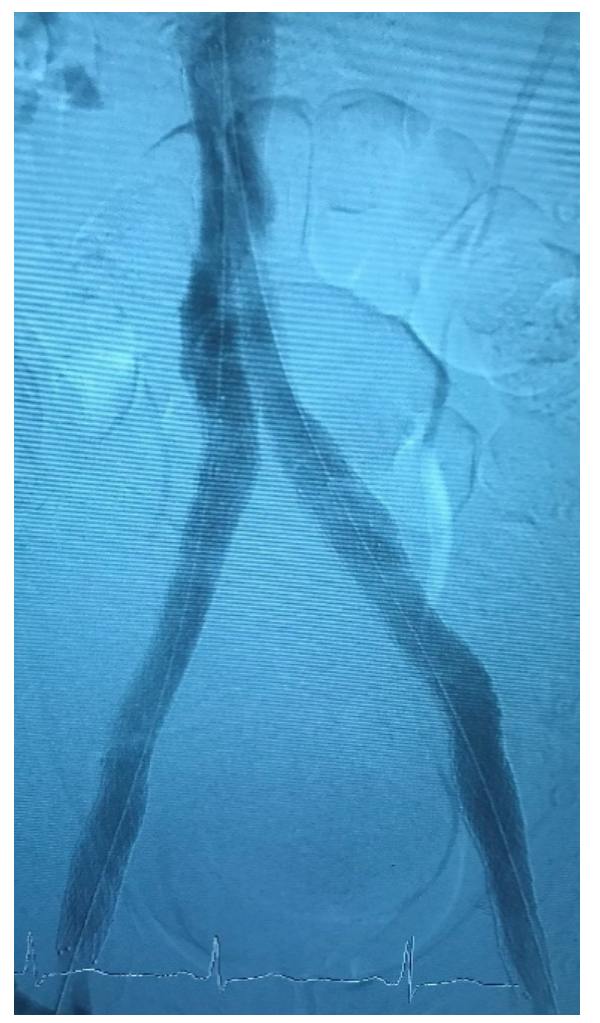

Figure 4. Final venogram showing patient bilateral iliac veins, IVC and IVC filter

\section{Discussion}

Patients with FVL are 11.4 times more likely to experience a thrombotic event than the general population [1]. Chronic iliocaval thrombosis has been reported in $3-30 \%$ of patients following IVC filter placement [2]. Thrombi in the IVC filter lead to decreased filter patency and lower extremity venous return, in turn, promoting stasis and increasing the risk of DVTs caudally [3]. It is difficult to delineate successfully trapped lower extremity embolus from in-situ thrombus within the filter [4]. Patients with both FVL and an IVC filter would theoretically be at a greater risk for IVC filter obstruction that could then lead to thrombosis caudally. Thus, both causes warrant investigation in a patient with recurrent thrombotic events.

Endovascular stenting for chronic iliocaval thrombosis has a published technical success rate - defined as successful recanalization and inline flow - of $100 \%$ in patients with $(\mathrm{n}=120)$ and without $(n=69)$ IVC filter associated disease [2,5]. High clinical success rate, although defined heterogeneously, has also been documented for this intervention. A decrease in CEAP score of at least one was reported in $84 \%$ of patients at 2 weeks and in $96 \%$ of patients at 6 months [2]. McDevitt et al. [5] also defined clinical success as a 1-point decrease in CEAP classification and stent patency was reported at 2-week, 6-month, 12-month, and 24-month with clinical success rate of $76 \%$, $85 \%, 87 \%$ and $100 \%$, respectively. Complete relief of pain was reported by $85 \%$ of 39 patients who underwent bilateral stenting for iliocaval obstruction [6] whereas "considerable improvement in lower extremity swelling/or pain that lasted at least 3 days or until hospital discharge" was documented in $78 \%$ of 18 symptomatic limbs [7]. It is interesting to note that clinical success in some studies increased as time passed. McDevitt et al. speculate that this may be due to augmentation of collaterals [5].
Patency rates have also been shown to be high post-endovascular stenting in the setting of both iliocaval obstruction and IVC filterassociated thrombosis. Chick et al. [2] reported primary iliocaval stent patency rates of $96.4 \%, 94.8 \%$ and $87.2 \%$, primary-assisted iliocaval stent patency rates of $98.2 \%, 97.0 \%$ and $90.3 \%$ and secondary iliocaval stent patency rates of $100 \%, 98.1 \%$ and $94.2 \%$ at 6-month, 12 -month and 24-month follow-ups, respectively, with $95 \%$ of the patients receiving Wallstent endoprostheses. McDevitt et al. [5] reported estimated primary patency rates of $91 \%, 88 \%$ and $62 \%$, primary-assisted patency rates of $98 \%, 95 \%$, and $81 \%$ and secondary-assisted rates of $100 \%, 100 \%$ and $100 \%$ at 6,12 and 24 month follow-ups with the majority of the patients receiving either Wallstent only $(n=53,77 \%)$ or Wallstent and Z-Stent ( $n=9,13 \%)$, endoprostheses.

Complications associated with endovascular stenting in the setting of both iliocaval thrombosis and IVC filter obstruction are infrequent. Both the 60-day mortality rate and 24-month attributable mortality rates are $0 \%[2,5]$. Chick et al. [2] reported $6(5 \%)$ minor complications and 2 (2\%) major complications in 120 patients during the perioperative period. McDevitt et al. [5] reported a 30-day mortality rate of zero, 90 -day mortality rate of $1.4 \%$ and 24 -month mortality rate of $10 \%$ with none of the deaths being related to the iliocaval stent reconstruction. However, complication rates can be higher for patients with thrombophilia given the inherent risk of recurrent thrombosis. While investigating stents placed in the lower extremity venous outflow tract, Neglen and Raju observed in-stent restenosis greater than $20 \%$ in $66 \%$ of patients with thrombophilia as opposed to $42 \%$ of patients without thrombophilia $(\mathrm{P}=.0005)$ [8]. This finding was corroborated by Titus et al. [9] who found that patients with thrombophilia were more likely to have worse outcomes than patients with idiopathic occlusion following iliofemoral stenting for venous occlusive disease. On the contrary, Blanch Alerany et al. [10] found no statistically significant difference in the rates of in-stent stenosis in patients with thrombophilia and those without thrombophilia. In fact, a later study of long-term stent-related outcomes in in chronic venous disease by Neglen and Raju concluded that thrombophilia was not an independent risk factor for stent occlusion $[11,12]$. Further studies are needed to accurately assess the long-term outcomes in patients with FVL and other thrombophilia's undergoing iliocaval reconstruction and other endovascular procedures for chronic thrombotic occlusion.

\section{Conclusion}

Patients with history of FVL, IVC filter placement and recurrent DVTs should be screened for IVC filter-associated thrombosis. Iliocaval obstruction secondary to IVC filter-associated thrombosis can be recanalized with a high degree of success using endovascular stenting. The risk-benefit ratio should be carefully considered before placing IVC filters in patients with FVL as these patients have a higher incidence of thrombotic events and may have worse outcomes following endovascular stenting. Further studies are required to determine longterm effects of iliocaval reconstruction in patients with FVL.

\section{References}

1. Simone B, De Stefano V, Leoncini E, Zacho J, Martinelli I, et al. (2013) Risk of venous thromboembolism associated with single and combined effects of Factor V Leiden, Prothrombin 20210A and Methylenetethraydrofolate reductase C677T: a meta-analysis involving over 11,000 cases and 21,000 controls. Eur J Epidemiol 28: 621-647. [Crossref]

2. Chick JF, Jo A, Meadows JM, Abramowitz SD, Khaja MS, et al. (2017) Endovascular iliocaval stent reconstruction for inferior vena cava filter-associated iliocava thrombosis: approach, technical success, safety, and two-year outcomes in 120 patients. J Vasc Interv Radiol 28: 933-939. [Crossref] 
Mohammad KO (2020) Iliocaval stent reconstruction following inferior vena cava filter associated chronic thrombotic occlusion in a patient with factor V leiden mutation - A case report and review of literature

3. Hajduk B, Tomkowski WZ, Malek G, Davidson BL (2010) Vena Cava Filter Occlusion and Venous Thromboembolism Risk in Persistently Anticoagulated Patients: A Prospective, Observational Cohort Study. Chest 137: 877-882. [Crossref]

4. L Duffet, M Carrier (2017) Inferior Vena Cava Filters. J Thromb Haemost 15: 3-12. [Crossref]

5. McDevitt J, Srinivasa RN, Hage AN, Bundy JJ, Gemmete JJ, et al. (2019) Tota endovenous recanalization and stent reconstruction for naïve non-inferior vena cava filter associated chronic iliocaval occlusive disease: Placement of 352 venous stents in 69 debilitated patients. Vasc Med 24: 1-10. [Crossref]

6. Neglén P, Darcey R, Olivier J, Raju S (2010) Bilateral stenting at the iliocaval confluence. J Vasc Surg 51: 1457-1466. [Crossref]

7. Vedantham S, Vesely TM, Parti N, Darcy MD, Pilgram TK, et al. (2003) Endovascular recanalization of the thrombosed filter-bearing inferior vena cava. $J$ Vasc Interv Radiol 14: 893-903. [Crossref]
8. Neglen P, Raju S (2004) In-stent recurrent stenosis in stents placed in the lower extremity venous outflow tract. J Vasc Surg 39: 181-187.

9. Titus JM, Moise MA, Bena J, Lyden SP, Clair DG (2011) Iliofemoral stenting for venous occlusive disease. J Vasc Surg 53: 706-712. [Crossref]

10. Blanch Alerany M, Izquierdo Lamoca LM, Ramirez Ortega M, Rivas IL, Desboeufs RZ, et al. (2014) Endovascular treatment of iliofemoral chronic post-thrombotic venous flow obstruction. J Vasc Surg Venous Lymphat Disord 2: 2-7. [Crossref]

11. Neglén P, Hollis KC, Olivier J, Raju S (2007) Stenting of the venous outflow in chronic venous disease: Long-term stent-related outcome, clinical, and hemodynamic result. $J$ Vasc Surg 46: 979-990. [Crossref]

12. Rollo JC, Farley SM, Jimenez JC, Woo K, Lawrence PF, et al. (2017) Contemporary outcomes of elective iliocaval and infrainguinal venous intervention for postthrombotic chronic venous occlusive disease. J Vasc Surg Venous Lymphat Disord 5 789-799. [Crossref]

Copyright: (C)2020 Mohammad KO. This is an open-access article distributed under the terms of the Creative Commons Attribution License, which permits unrestricted use, distribution, and reproduction in any medium, provided the original author and source are credited. 